\title{
Case Report: Description of two fractures during the use of a powered exoskeleton
}

\author{
F. H. M. van Herpen ${ }^{1}$ - R. B. van Dijsseldonk $\mathbb{B}^{2,3} \cdot$ H. Rijken ${ }^{1}$ - N. L. W. Keijsers ${ }^{2,3}$ - J. W. K. Louwerens ${ }^{4} \cdot$ \\ I. J. W. van Nes ${ }^{1}$
}

Received: 23 September 2019 / Revised: 29 October 2019 / Accepted: 30 October 2019

(C) International Spinal Cord Society 2019

\begin{abstract}
Introduction Powered robotic exoskeletons are a promising solution to enable standing and walking in patients with spinal cord injury (SCI). Although training and walking with an exoskeleton in motor complete SCI patients is considered safe, the risks of unexpected (technical) adverse events and the risk of fractures are not fully understood. This article reports the occurrence of two different cases of bone fracture during exoskeleton usage. Furthermore, advice is given for extra safety training and instructions.

Case presentation The first case concerns a 47-year-old woman with T12 AIS A SCI. Her exoskeleton shut down unexpectedly probably causing a misalignment of the joints of her lower extremities relative to the joints of the exoskeleton, which resulted in a fracture of her left tibia. The second case involves a 39-year-old man with L1 AIS B SCI. An unexpected fracture of the right distal tibia occurred without a specific prior (traumatic) incident.

Discussion Exoskeleton training instructors, SCI patients and their buddies should be instructed how to handle emergency situations. Furthermore, they should be aware of the risk of stress fractures of the lower extremities. Proper alignment of the exoskeleton relative to the body is of utmost importance to reduce fracture risk. In the case of swelling and discoloring of the skin, radiographic examination should be performed in order to exclude any fracture.
\end{abstract}

\section{Introduction}

New technological developments, like a powered robotic exoskeleton, make it possible for people with motor complete spinal cord injury (SCI) to independently stand, walk,

Supplementary information The online version of this article (https:// doi.org/10.1038/s41394-019-0244-2) contains supplementary material, which is available to authorized users.

$\triangle$ F. H. M. van Herpen

f.vanherpen@maartenskliniek.nl

1 Department of Rehabilitation, Sint Maartenskliniek, Nijmegen, The Netherlands

2 Department of Research, Sint Maartenskliniek, Nijmegen, The Netherlands

3 Department of Rehabilitation, Donders Institute for Brain, Cognition and Behaviour, Radboud University Medical Center, Nijmegen, The Netherlands

4 Department of Orthopaedics, Sint Maartenskliniek, Nijmegen, The Netherlands and climb stairs. Nowadays, multiple commercial exoskeletons are available and some of these exoskeletons are certified for use outside a clinical setting. In general, the training programs in a clinical setting with an exoskeleton are considered feasible [1-4]. The use of these powered exoskeletons in real-world settings has also shown to be safe [2]. However, literature about adverse events involving a powered exoskeleton is scarce. The adverse events that have been described are fractures and skin aberrations [2, 46]. Two incidents of bone fractures with exoskeleton use have been reported in the literature [4,5]. A meta-analysis by Miller et al. [2] based on one single reported event, determined an incidence of bone fracture at any time during the training program of $3.4 \%$.

Our institute has offered the ReWalk ${ }^{\mathrm{TM}}$ in-hospital training program since 2015 , as it was the only CE and FDA approved exoskeleton for both in-hospital and home use [7]. The program consists of three $1.5 \mathrm{~h}$ training sessions a week for 8 weeks. These training sessions are under the supervision of at least two physiotherapists, who are certified exoskeleton instructors. When the participants are able to use the exoskeleton independently and safely and 
have sufficient exoskeleton skills, the participants are enabled to use the exoskeleton in their homes for an additional 2 weeks [7]. In case of home use, a 'buddy' is instructed by the therapists to guide a patient walking in an exoskeleton and the participants are taught to always be accompanied by this instructed buddy.

This article reports two cases of bone fractures during exoskeleton usage: one during home use and one during an in-hospital training session. Subsequently, advice is given for additional training and instructions regarding safety procedures to prevent these adverse events.

\section{Case presentation}

\section{Case 1}

The first case concerns a 47-year-old woman with a traumatic T12 AIS A traumatic SCI since 2004. She did not have spasticity or contractures in the joints of her lower extremities. She completed the training program successfully along with 2 weeks of exoskeleton use at home. After completing the training program and still being enthusiastic about the exoskeleton, ReWalk allowed her to use the exoskeleton on several occasions such as demonstrations, presentations, and occasionally at home. When walking with the exoskeleton, she was always assisted by her buddy.

During at home usage, while walking outside, she lost her balance. Her buddy grasped the exoskeleton to help her restore her balance and accidentally pressed the power button of the exoskeleton. As a consequence of the sudden loss of power (i.e., in the case of a major system failure or turning the exoskeleton off while in standing position), the ReWalk $^{\mathrm{TM}}$ exoskeleton ran the 'graceful collapse' algorithm and gently lowered the woman to the floor.

While she was on the floor, the woman was guided by her buddy to the sitting position and she gave the command to stand up again with her remote-control input device. While standing up, she heard a snapping sound in her left calf and the system stopped unexpectedly halfway and began to collapse again to the sitting position. With help of her buddy, she detached herself from the exoskeleton and sat in her wheelchair. She noticed a hematoma on her left knee and back along with unnatural mobility of her left knee.

Radiographic examination revealed severe osteopenic bone with a comminuted intra-articular fracture of the left tibial plateau. The orthopedic surgeon performed closed reduction followed by treatment with plaster cast for 8 weeks. This was complicated by pressure ulcers on her heel. Full consolidation of bone was established not until 9 months after trauma. After healing of the bone she returned to walking with her exoskeleton without problems.

Evaluation of the exoskeleton by the manufacturer, did not reveal any deficits in the hard- or software of the exoskeleton. Our hypothesis for the bone fracture, is that the position of her left lower extremity was misaligned relative to the joints of the exoskeleton due to the graceful collapse (Fig.1). It is likely that the subsequent standing up motion caused relatively excessive torsion forces on her lower extremities due to this misalignment, resulting in a comminuted intra-articular fracture of the left tibial plateau.

After the incident, a dual energy x-ray absorptiometry (DEXA)-scan was performed which showed a $T$-score of her left hip of -2.7 and of her right hip of -2.7 , indicating osteoporosis. Treatment with additional calcium, vitamin D, and bisphosphonate was started.

Case 2

The second case concerns a 39-year-old man with L1 AIS B traumatic SCI since 2000. He started exoskeleton training at the beginning of 2018. He did not have spasticity or contractures in the joints of his legs. During his fifth inhospital training session he noticed some swelling and experienced slight pain at his right ankle. No incidents had occurred during the training session or at a former training session. The pain subsided the day after the fifth session. Two days later he again noticed an increase of the swelling and a bluish-red discoloration of the skin. An emergency physician considered this to be an infection and prescribed antibiotics. After the weekend, he visited his own physician and radiography revealed a fracture of the distal tibia at the tibial plafond with damage of the subchondral bone. The cause of trauma was discussed in a multidisciplinary team meeting with the physiatrist, orthopedic surgeon, and physiotherapists. No incident or specific movement that could have caused the fracture was noticed or recalled.

A DEXA-scan was performed and revealed a $T$-score of his left hip of -1.2 and of his spine (L4) of 1.2, indicating

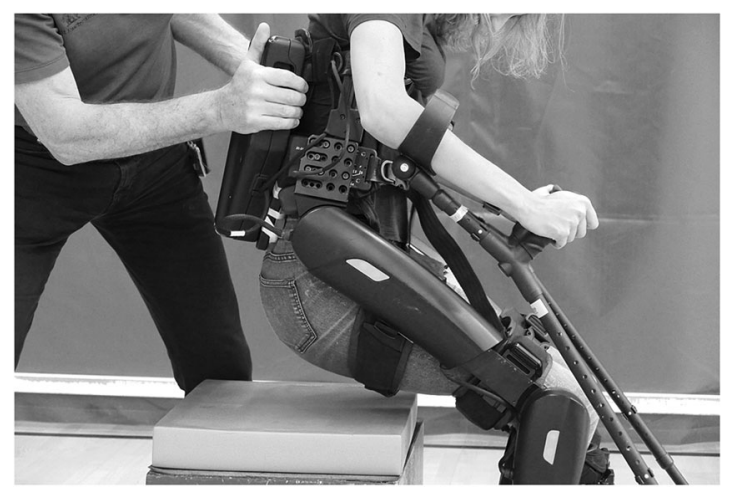

Fig. 1 Example of misalignment of the hip joint relative to the exoskeleton 
no osteoporosis or osteopenia of these specific bones. However, the conventional radiography showed a relative osteoporotic distal tibia and the individual received the advice to start with vitamin $\mathrm{D}$.

\section{Discussion}

Although training with an exoskeleton has generally been shown to be safe [2], the present cases show that there is always a risk of unexpected serious adverse events. A metaanalysis by Miller et al. [2] reported a 3.4\% incidence of bone fracture in exoskeleton-assisted walking. This low incidence rate was based on a single reported bone fracture in one study in the literature. The two additional cases reported in the current study and a case reported by Gagnon et al. [4] suggest that the $3.4 \%$ incidence might be an underestimation.

Loss of bone mass coupled with excessive torque of the exoskeleton on the lower extremities during exoskeletonassisted walking and the potential risk of misalignment, are associated with a higher risk of bone fractures [8]. We have described two cases of bone fractures related to exoskeleton use, one at home use after a successful in-hospital training and one during an in-hospital training period.

In the first case, the woman and her buddy completed the training program successfully and were very experienced in using the exoskeleton. However, they were not trained well enough in reacting to situations in which the exoskeleton would shut down unexpectedly. During an unexpected shut down, the ReWalk ${ }^{\mathrm{TM}}$ exoskeleton will run a 'graceful collapse' algorithm that gently lowers the user to the floor. The first case made us realize that both the individual with the SCI and their buddy need extra instructions on how to react in such an emergency situation. Ever since this incident, we have extended our exoskeleton training protocol with extra training and instructions regarding safety procedures in case of unexpected software problems or in the case the exoskeleton is accidentally switched off. A storyboard after accidental shut down of the exoskeleton is shown in Fig. 2; and the instructions are available in the Supplementary Video.

After accidently switching off an exoskeleton the individual should switch the exoskeleton back on as fast as possible, resulting in a lock of the ReWalk ${ }^{\mathrm{TM}}$ exoskeleton in its current position. Second, the buddy must stabilize the standing position of the user and press the power button again (switch off) to initiate the 'graceful collapse' algorithm, guiding the user to the floor or to a nearby chair, if present. Lastly, when the user is in a stable position, the user is assisted out of the exoskeleton and is instructed not to use the device until further inspection.

One of the main causes for a fracture of the lower extremities during exoskeleton use, is misalignment of the joints of the exoskeleton relative to the joints of the lower extremities, especially in osteopenic or osteoporotic bones. In our first case, the hip joints of the exoskeleton were misaligned relative to the user's own hip joints after the 'graceful collapse' motion (Fig.1). The subsequent standing up motion caused excessive torsion forces on her legs, resulting in the fracture of her left tibial plateau. Proper alignment of the exoskeleton relative to the body is, therefore, of the utmost importance. Alignment should be checked before and after the sit-to-stand transition and always after a graceful collapse motion.

Loss of bone mass and osteoporosis are common secondary complications associated with SCI with increased risk of bone fractures [9]. In the first case, excessive torsion forces on the osteoporotic bones of the lower extremities resulted in the fracture of the tibial plateau. In the second case the bone fracture occurred during one of the training sessions. The participant was the 18th patient that was trained in our rehabilitation center. The training sessions are always given by the same three ReWalk ${ }^{\mathrm{TM}}$ certified physiotherapists, who had given more than $600 \mathrm{~h}$ of training sessions, indicating they were very experienced. Neither the participant, nor the physiotherapists noticed an incident during or after the training session. Furthermore, no
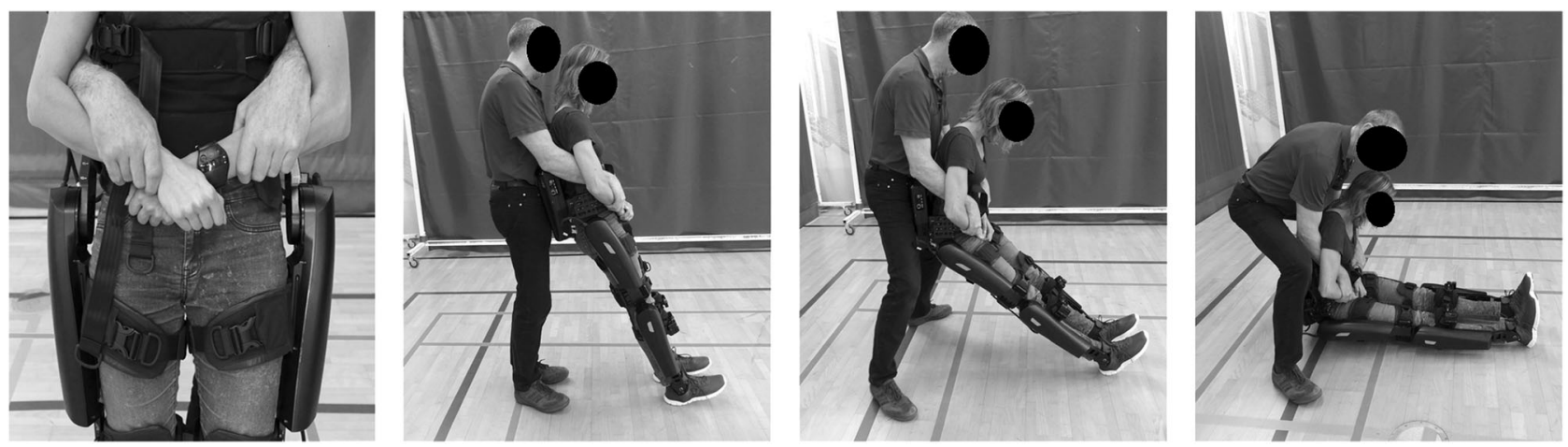

Fig. 2 Storyboard of the training instructions after accidental shut down of the exoskeleton 
misalignment of the exoskeleton was recalled by the physiotherapists. To our knowledge, the only logical explanation of the second case was axial compression of the relatively dense talus on the osteoporotic distal tibia, resulting in a fracture of the distal tibia. It is important to be aware of complications as a result of relatively osteoporotic or osteopenic bones of the lower extremities. Gagnon et al. [4] reported an individual with bilateral type 1 nondisplaced fractures of the calcaneus after two familiarization sessions and the first training session with the EKSO ${ }^{\mathrm{TM}}$ robotic exoskeleton. Benson et al. [5] reported an individual with a hairline fracture of the talus seen on magnetic resonance imaging (MRI). They reported that, apart from a temporarily swollen ankle noticed the morning following the fourth training session, no other signs and symptoms were present. Similar to our second case, uncertainties about the specific cause of the fracture existed in the studies of Gagnon et al. and Benson et al. The limited clinical symptoms reported by Benson et al. show many similarities with our second case. Therefore, we recommend checking the lower extremities after each training session. In the case of swelling or discoloring of the skin, X-ray examination should be performed to exclude fracture. In case of persistent symptoms, repeat X-ray or other additional examination (like MRI) should be performed because stress fractures are not always present at first examination.

Some studies involving powered exoskeletons screen for osteoporosis to define objective exclusion criteria $[2,6]$. However, criteria differ between studies and the association between fracture risk at specific levels of bone mass density is still unknown [6]. Therefore, cut-off points with respect to bone mass density or a threshold of exclusion for exoskeleton usage, are difficult to define. After the incidents in our hospital, ReWalk advised to perform a DEXA-scan before commencing a training program of each individual patient. ReWalk recommends a $T$-score of $>-3.5$. In our cases, both patients had $T$-scores above $-3.5(-2.7$ and -1.2 respectively), but still sustained fractures of the lower extremities.

\section{Conclusion}

Although training and walking with an exoskeleton in motor complete SCI patients is considered safe, one should always be aware of risks of unexpected (technical) adverse events and the risk of fractures. It is important to incorporate specific instructions regarding safety procedures for unexpected situations in an exoskeleton training program to prevent adverse events. The higher risk of (stress) fractures, especially in the case of osteopenic or osteoporotic bones, should be always considered. Maintaining proper alignment of the exoskeleton relative to the body is of the utmost importance and regular checks are necessary. Powered robotic exoskeletons are a promising solution to enable standing and walking in patients with SCI; however we strongly recommend training users in a multidisciplinary setting including experienced physiotherapists, a physiatrist and a researcher so as to gain more experience in the possibilities of these great innovations.

Acknowledgements The exoskeleton training of the physiotherapists by ReWalk Robotics was given before the start of the study. ReWalk Robotics did not have any influence on the entire study.

\section{Compliance with ethical standards}

Conflict of interest The authors declare that they have no conflict of interest.

Publisher's note Springer Nature remains neutral with regard to jurisdictional claims in published maps and institutional affiliations.

\section{References}

1. Zeilig G, Weingarden H, Zwecker M, Dudkiewicz I, Bloch A, Esquenazi A. Safety and tolerance of the ReWalk ${ }^{\mathrm{TM}}$ exoskeleton suit for ambulation by people with complete spinal cord injury: a pilot study. J Spinal Cord Med. 2012;35:101-96.

2. Miller LE, Zimmermann AK, Herbert WG. Clinical effectiveness and safety of powered exoskeleton-assisted walking in patients with spinal cord injury: systematic review with meta-analysis. Med Devices. 2016;9:455-66.

3. Adriaansen JJ, van Asbeck FW, van Kuppevelt D, Snoek GJ, Post MW. Outcomes of neurogenic bowel management in individuals living with a spinal cord injury for at least 10 years. Arch Phys Med Rehabil. 2015;96:905-12.

4. Gagnon DH, Escalona MJ, Vermette M, Carvalho LP, Karelis AD, Duclos $\mathrm{C}$, et al. Locomotor training using an overground robotic exoskeleton in long-term manual wheelchair users with a chronic spinal cord injury living in the community: Lessons learned from a feasibility study in terms of recruitment, attendance, learnability, performa. J Neuroeng Rehabil. 2018;15:12.

5. Benson I, Hart K, Tussler D, van Middendorp JJ. Lower-limb exoskeletons for individuals with chronic spinal cord injury: findings from a feasibility study. Clin Rehabil. 2016;30:73-84.

6. Mekki M, Delgado AD, Fry A, Putrino D, Huang V. Robotic rehabilitation and spinal cord injury: a narrative review. Neurotherapeutics. 2018;15:604-17.

7. van Dijsseldonk RB, Rijken H, van Nes IJW, van de Meent H, Keijsers NLW. A framework for measuring the progress in exoskeleton skills in people with complete spinal cord injury. Front Neurosci. 2017;11:1-12.

8. He Y, Eguren D, Luu TP, Contreras-Vidal JL. Risk management and regulations for lower limb medical exoskeletons: a review. Med Devices Evid Res. 2017;10:89-107.

9. Bauman WA. Risk factors for osteoporosis in persons with spinal cord injury: what we should know and what we should be doing. J Spinal Cord Med. 2004;27:212-3. 